\title{
Concept of Virtual Incision for Minimally Invasive Surgery
}

\author{
Yuki Horise, Atsushi Nishikawa, Toshikazu Kawai, \\ Ken Masamune and Yoshihiro Muragaki
}

Additional information is available at the end of the chapter

http://dx.doi.org/10.5772/intechopen.68561

\begin{abstract}
Minimally invasive surgery has been introduced to various surgical fields for its benefits such as smaller scars and less pain as compared to open surgery. Highly skilled surgical techniques are required for surgeons to conduct minimally invasive surgery with fewer ports, whereas minimally invasive surgery has a number of advantages for patients. Singleincision laparoscopic surgery (SILS), in which surgical instruments and a laparoscope are inserted through a single port, has better cosmetic results than conventional multi-incision surgery; moreover, the scar is invisible when the port is opened in navel. However, instrument collisions and visual defects often occur due to the limited space of the single opening. We propose a new surgical approach entitled "virtual incision" that enables surgeons to increase the number of openings virtually. Using our approach, we have developed two types of master-slave surgical robot systems for SILS-remote-operated and local-operated systems - which have operability close to that of multiple-incision surgery. Through evaluation of these systems, we demonstrated that the visual field and operability during virtual incision surgery are similar to those of conventional multi-incision surgery. Our surgical approach can be applied to not only single-incision surgery but also multiincision surgery, and is very likely to improve operability.
\end{abstract}

Keywords: master-slave, robot, surgery, virtual incision

\section{Introduction}

In the past, curing illness was taken to be the highest priority and surgeons did not pay equal regard to patients' quality of life (QOL). Recently, however, as medical technology has progressed, equal importance can be given to both. Minimally invasive treatment has been 
introduced in various medical fields to reduce the patient's psychic and physical burden. For example, intravascular treatment with a catheter has been used in cerebrovascular, cardiovascular and peripheral artery surgeries, etc., and the patient's burden is drastically decreased [1]. Particle radiotherapy and advanced radiotherapy can lessen the side effects and emphasize the therapeutic effects more than conventional radiation therapy [2]. Laparoscopic surgery is a kind of minimally invasive endoscopic surgery that targets the digestive organs and requires multiple small ports to insert long surgical instruments and a cylindrical camera called a 'laparoscope'. Laparoscopic techniques bring many benefits to patients, such as smaller scars, fewer complications, and shorter hospitalization than conventional open surgery $[3,4]$. To achieve less invasiveness, a new surgical methodsingle-incision laparoscopic surgery (SILS) - has been developed. In this method, surgical instruments, including a laparoscope, are inserted through a single opening. SILS is more cosmetic than general laparoscopic surgery since the number of ports is reduced from many to one. It is sometimes called "scar-less surgery" because the scar is invisible when the port is made at navel [5-7]. However, SILS requires high levels of skill for operating surgeons due to physical challenges such as collisions between instruments and visual defects caused by the single opening [8-10].

Minimally invasive surgery places a large burden on operating surgeons but brings great benefits for patients and preserves their QOL. To address this matter, various systems for minimally invasive surgery have been developed. The da Vinci (Intuitive Surgical Inc., CA, USA) is one of the most famous master-slave surgical robot systems in the world and can conduct laparoscopic surgery remotely with the operability close to that of open surgery by offering a 3D visual field, precise manipulation, and hand tremor cancellation. Although this robot was originally developed to support conventional multi-port laparoscopic surgery, it has also been used in single-port surgery due to its high functionality [11, 1]. Yet, it has mechanical problems such as arm collision and takes a long time to learn, even for skilled surgeons [13].

To achieve SILS that satisfies both patients and surgeons, we propose a new surgical concept called "virtual incision" and have evaluated the effectiveness of our concept by two types of master-slave robot systems for SILS.

\section{Virtual incision}

In SILS, the same surgical instruments and laparoscope are used as in conventional laparoscopic surgery, and the number of surgical incisions is the only difference between these operating methods. The fact that the number of surgical incisions has decreased from 3 to 1, as shown in Figure 1(a) and (b), is one cause of the technical problems in SILS such as instruments collisions and visual defects. Thus, we propose a new surgical approach entitled "virtual incision" in which the number of ports is increased not physically but virtually. This concept provides SILS the operability close to that of multi-port surgery. 


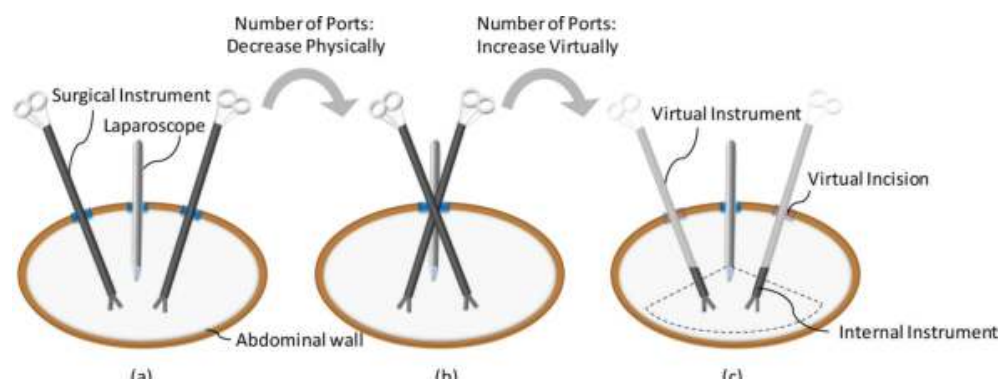

(a)

(b)

(c)

Figure 1. The number of surgical incisions required; (a) conventional multi-incision surgery, (b) single-incision laparoscopic surgery (SILS), and (c) SILS with two virtual incisions.

Making a "virtual incision" requires two conditions: (1) internal surgical instruments must behave the same as conventional laparoscopic surgery within the range of a laparoscopic view as shown in Figures 1(c) and (2) the operator must hold other external instruments to control the internal surgical instruments and manipulate them in the same way as conventional laparoscopic surgery. Please note that the operators normally look not at their hands but at a laparoscopic view displayed on a monitor during surgery. If these two conditions are satisfied, two virtual incisions are created on the abdominal wall in the direction of the long axis of the internal surgical instruments seen on the laparoscopic view. Therefore, surgeons can conduct SILS as if they were operating multi-port surgery even though there is only a single real port in the abdominal wall.

\section{Master-slave robot systems using the concept of virtual incision}

We have developed two types of master-slave robot systems using our proposed "virtual incision" approach: a remote-operated system which requires two virtual incisions, achieving three-incision surgery [14] and a local-operated system which requires one virtual incision, achieving two-incision surgery [15].

\subsection{Remote-operated master-slave robot system}

\subsubsection{System configuration}

A remote-operated master-slave robot system is mainly composed of two master instruments, two slave bending instruments and a control PC. We use two normal surgical instruments as master, which are inserted through two incisions at the tip of two holding arms set on a table as shown in Figure 2. A small magnetic sensor (3D-Guidance, Ascension Technology Corporation, VT, USA) is attached to each tip of the master instrument to measure the tip's position and posture. On the slave side, the two flexible instruments and a laparoscope are attached to commercial robotic arms (slave part of ZEUS surgical robot system, Computer 


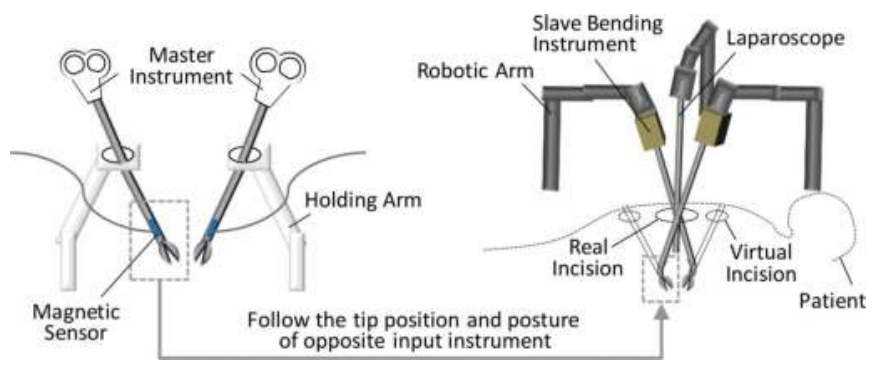

Figure 2. Correspondence between master instruments and flexible slave instruments.

Motion Inc., CA, USA), and they are all inserted into a real single opening in the patient's abdominal wall. The two slave instruments are crossed through the port and the tip of these instruments can be bent with a wire-driven mechanism. Each master instrument corresponds to the opposite slave instrument; that is, the left-side master instrument controls the slave instrument attached to right-side robotic arm; thus, the instrument tip of master behaves in the same way as that of slave in the area of the dashed square in Figure 2. Although the motion ratio between master and slave is adjustable (e.g., 5:1 to achieve a precise motion on the slave side), in our robot system we set the motion ratio of $1: 1$ to provide the surgeons the same operability as conventional laparoscopic surgery.

In this case, our proposed remote-operated master-slave robot system meets the requirements for the concept of virtual incision, then two virtual incisions are created in the abdominal wall in the long axis direction of the distal part of the slave instruments, which means that the operating surgeon can conduct SILS with three ports (one real and two virtual).

\subsubsection{System evaluation}

\subsubsection{Operating conditions}

Prior to system evaluation, we prepared an imitation of conventional operating conditions (multi-port and single-port surgery) by adjusting the set-up of master and slave, such as the number of ports and the shape of instruments, to compare our proposed method with conventional methods, as shown in Figure 3. In imitated multi-port surgery (iMPS), there are two surgical ports on the master and slave sides, and the distal part of the two slave bending instruments is set linear shape. Each master instrument corresponds to the slave instrument on the same side; thus, the same operability as in conventional multi-port surgery can be achieved. In imitated single-port surgery (iSPS), the distal parts of the two holding arms are moved close to each other on the master side, and the two slave instruments are crossed and pass through one incision, and the distal part of these is set linear shape on slave side. The master instruments correspond to the slave instruments as in the operating condition of iMPS, providing almost the same operability as conventional single-port surgery. In the proposed SILS, the configuration of and correspondence between the master and slave instruments are set as described in Section 3.1.1. 


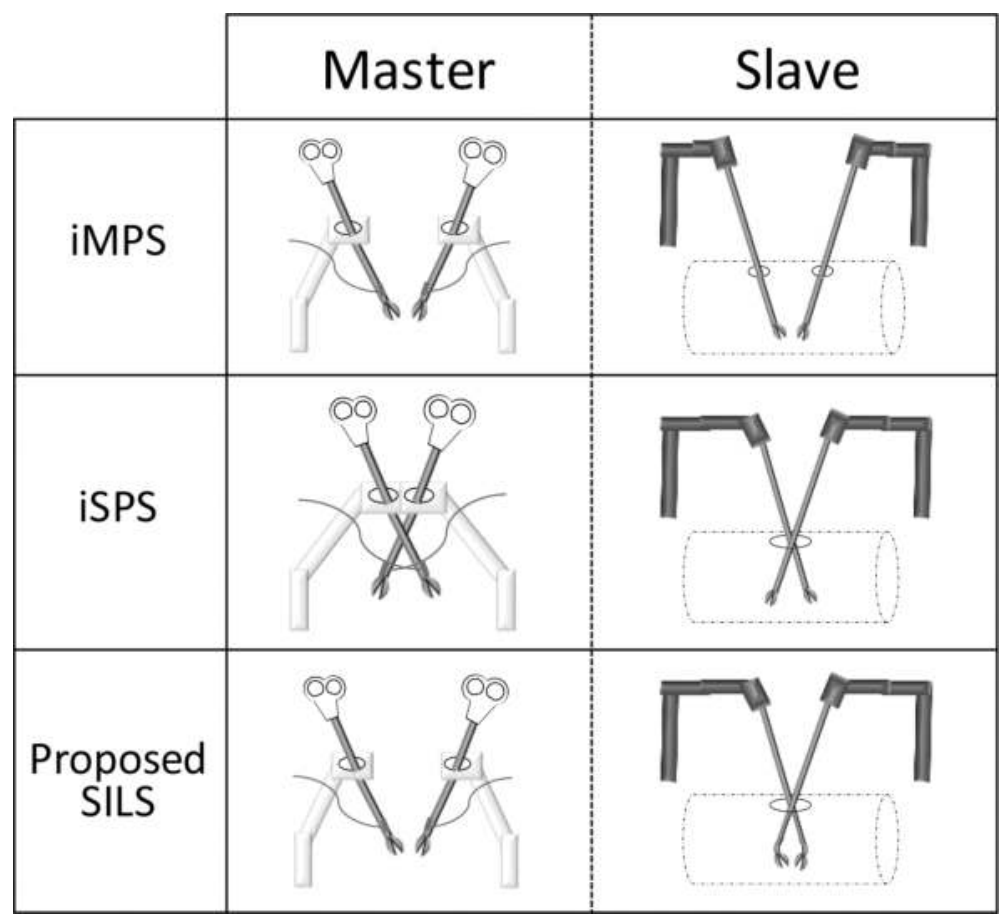

Figure 3. Master and slave configuration of three operating conditions: imitated multi-port surgery (iMPS), imitated single-port surgery (iSPS), and SILS with proposed remote-operated system (proposed SILS).

\subsubsection{Task}

In the remote-operated master-slave robot system, we conducted two experiments using (1) a simulated slave robot and (2) a real slave robot.

Experiment (1): a monitor is set behind the master, displaying an imaginary laparoscopic view as shown in Figure 4(a). Using this system, four gastral surgeons conducted an objectmoving task, in which the operator moves a series of balls around two boxes to each box by manipulating two master instruments, as indicated in Figure 4(b). One trial is set as moving a ball 40 times, and the surgeons implemented two trials each in three operating conditions (iMPS, iSPS, and proposed SILS).

Experiment (2): two robotic arms are mounted on a surgical bed and two bending slave instruments are attached to each arm. A box is placed on the surgical bed, and the two slave instruments and a laparoscope are inserted into the box, as shown in Figure 5. A monitor is set as well as in Experiment (1) and a real laparoscopic view is displayed. Under this experimental environment, another gastral surgeon conducted an object-touching task, in which the operator touches a cylindroid object in the box using the two slave instruments by manipulating the two master instruments. He conducted seven trials in each of the two operating conditions (iSPS and proposed SILS). 


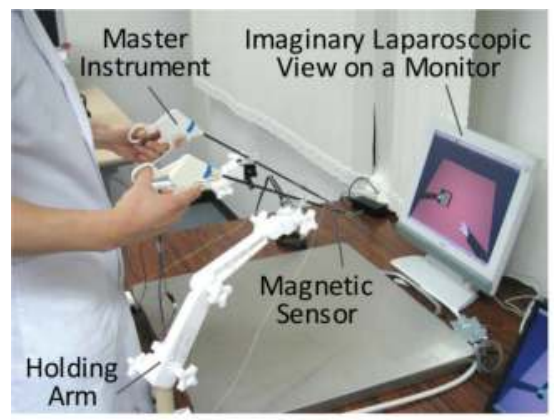

(a)

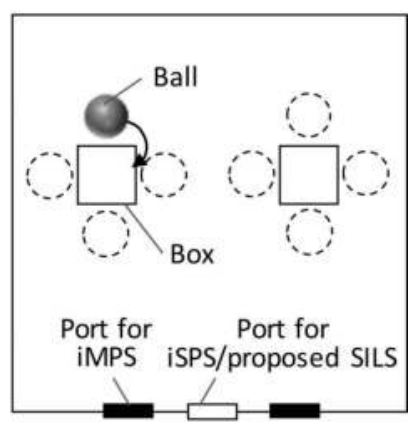

(b)

Figure 4. Object-moving task: (a) experimental set-up during the task in iMPS and (b) task detail; black port for iMPS and white port for iSPS and proposed SILS.

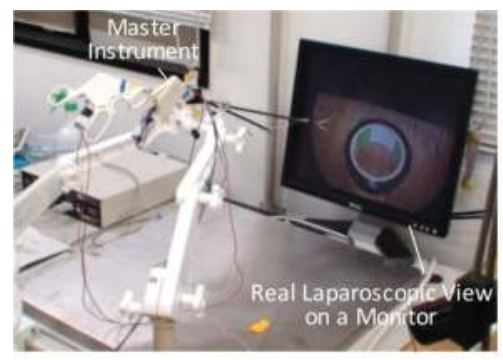

(a)

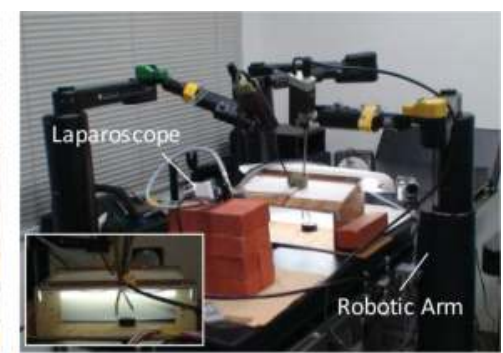

(b)

Figure 5. Object-touching task during the task in iSPS: (a) master set-up and (b) slave set-up.

\subsubsection{Result}

Experiment (1): average object-moving times among the four surgeons for each trial were 251 $\pm 61 \mathrm{~s}$ (iMPS), $310 \pm 52 \mathrm{~s}$ (iSPS), and $247 \pm 67 \mathrm{~s}$ (proposed SILS) in trial 1, and $182 \pm 29 \mathrm{~s}$ (iMPS), $248 \pm 77 \mathrm{~s}$ (iSPS), and $178 \pm 15 \mathrm{~s}$ (proposed SILS) in trial 2. There was no significant difference between iMPS and proposed SILS $(p=0.23>0.1)$, and a significant difference between iSPS and proposed SILS was confirmed $(p=0.011<0.05)$.

Experiment (2): average object-touching times were $25 \pm 7 \mathrm{~s}$ (iSPS) and $11 \pm 4 \mathrm{~s}$ (proposed SILS).

\subsection{Local-operated master-slave robot system}

\subsubsection{System configuration}

A local-operated master-slave robot consists of a master device, a slave robotic instrument, and a control PC. The master device is placed above the patient's abdominal wall and can 
be fixed to a surgical table using a conventional passive grasping holder. The slave robotic instrument is also attached to the surgical table with another grasping arm, and the robotic instrument can change between the two states of straight or bent; it is straight when passing through an incision and then is bent and set in alignment with the master device in the body as indicated in Figure 6(a). The master device and slave robotic instrument have five-DOF motion as in conventional laparoscopic surgery, including a grip/tip opening and closing (OC), spherical motion around the base point (pitch and yaw), axial rotation (roll), and extraction and contraction (EC) along the longitudinal axis. The posture and tip opening and closing of the slave robotic instrument have a point-symmetrical relationship with those of the master device, and the length of the slave instrument is inversely proportional to that of the master device. The master device and slave robotic instrument are moved simultaneously through the abdominal wall with a motion ratio of $1: 1$, thus they behave like a normal commercial surgical instrument inserted into a port. Our proposed system could be used as a substitute for the left-sided surgical instrument in SILS, especially in single-incision laparoscopic cholecystectomy as shown in Figure 6(b) since the left-sided surgical instrument is often used for grasping tissue.

In this case, our proposed local-operated master-slave robot system meets the requirements of virtual incision, and one virtual incision is created in the abdominal wall between the master device and the slave robotic instrument as illustrated in Figure 6(a). This means that the operating surgeon can conduct SILS with two ports (one real and one virtual).

\subsubsection{System evaluation}

\subsubsection{Task}

In the local-operated master-slave robot system, we conducted two experiments: (1) a basic experiment and (2) an ex vivo experiment using a porcine liver.

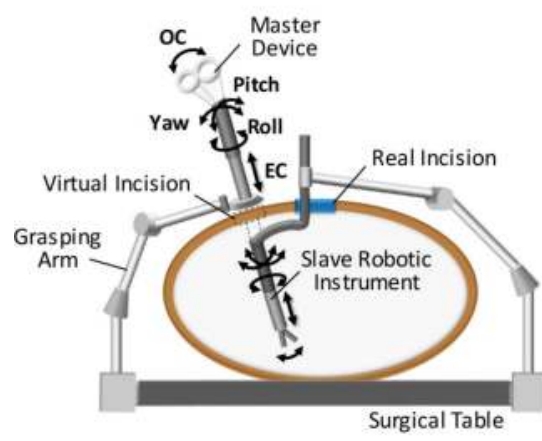

(a)

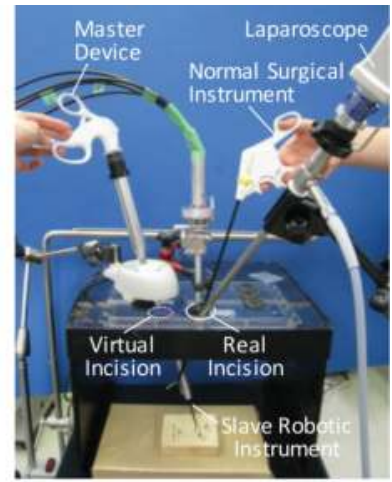

(b)

Figure 6. Local-operated master-slave robot system: (a) concept of proposed system and (b) surgical instrumental set-up for single-incision laparoscopic cholecystectomy. 
Experiment (1): a training box is placed on a surgical table, and a master device is set above the box as a left-side surgical instrument as shown in Figure 7(a). A right-sided normal surgical instrument, a laparoscope, and a slave robotic instrument are all inserted into the single real opening. The operator moves a series of rings placed on a peg board in the box from left to right using the surgical instruments with both hands while observing the laparoscopic view on a monitor. The ring is lifted from the left-side peg by the left-side instrument in the laparoscopic view, transferred to the opposite-sided instrument, and placed on the right-side peg, as illustrated in Figure 7(b). To evaluate the operability of our proposed SILS, we prepared two conventional SILS operating conditions-SILSc and SILSp-in which two normal surgical instruments are inserted through a single port and are manipulated with crossed and parallel set-ups, respectively. One trial is set as moving three rings three times, and three gastral surgeons (Surgeon A, B, and C) conducted three trials each under three operating conditions (SILSc, SILSp, and proposed SILS).

Experiment (2): the operating condition of proposed SILS is set as in Experiment (1), and a normal resection instrument is used as a right-sided instrument. As shown in Figure 7(c), a porcine liver with a gallbladder is fixed to a board in the training box, and the cystic duct has previously been clipped. In this experiment, we simulate a single-port laparoscopic cholecystectomy, and an operator separates the gallbladder from the liver by manipulating the surgical instruments with both hands while watching the laparoscopic view.

\subsubsection{Result}

Experiment (1): average switching time between the instruments and standard deviation of Surgeon A were $11.65 \pm 14.97 \mathrm{~s}$ (SILSc), $21.64 \pm 33.85 \mathrm{~s}$ (SILSp), and $7.03 \pm 2.49 \mathrm{~s}$ (proposed SILS); those of Surgeon B were $6.82 \pm 4.51 \mathrm{~s}$ (SILSc), $13.82 \pm 29.40 \mathrm{~s}$ (SILSp), and $8.48 \pm 3.58 \mathrm{~s}$ (proposed SILS); and those of Surgeon C were $10.04 \pm 5.07 \mathrm{~s}$ (SILSc), $35.82 \pm 34.66 \mathrm{~s}$ (SILSp),

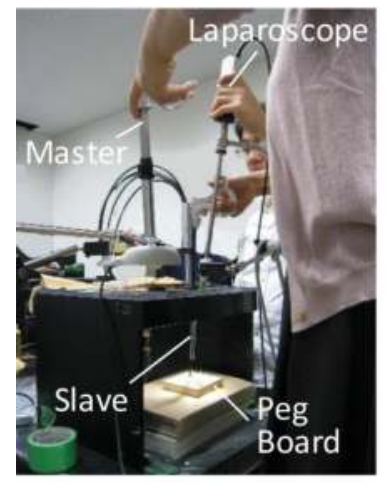

(a)

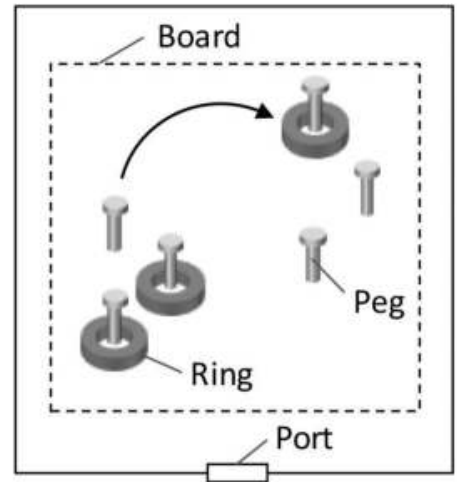

(b)

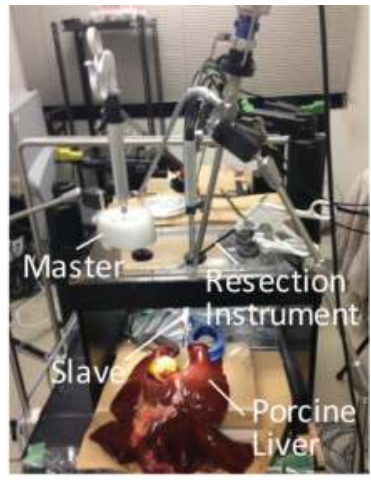

(c)

Figure 7. Evaluation experiment of local-operated master-slave robot system: (a) object-moving task in the operating condition of our proposed SILS, (b) peg board detail, and (c) ex vivo experimental set-up in single-port laparoscopic cholecystectomy. 
and $6.48 \pm 2.25 \mathrm{~s}$ (proposed SILS). The average switching times among the three surgeons were $9.50 \pm 9.70 \mathrm{~s}$ (SILSc), $22.25 \pm 33.57 \mathrm{~s}$ (SILSp), and $7.23 \pm 2.87 \mathrm{~s}$ (proposed SILS).

The number of collisions between the instruments in the box during the task of Surgeon A was 7 times (SILSc), 15 times (SILSp), and 0 times (proposed SILS); those of Surgeon B were 3 times (SILSc), 9 times (SILSp), and 1 time (proposed SILS); and those of Surgeon C were 1 time (SILSc), 18 times (SILSp), and 0 times (proposed SILS). The average number of collisions of all surgeons was 3.67 times (SILSc), 14 times (SILSp), and 0.33 times (proposed SILS). Meanwhile, the collision between the instrument and laparoscope occurred frequently outside the box under two conventional SILS operating conditions; however, the master device did not conflict with the laparoscope or the other instrument in our proposed SILS.

Experiment (2): the operator separated the gallbladder from the liver without instrument collision, grasping it using the slave robotic instrument by manipulating the master device with the left hand and cutting it using the resection instrument with the right hand.

Laparoscopic views during Experiments (1) and (2) are presented in Figure 8.

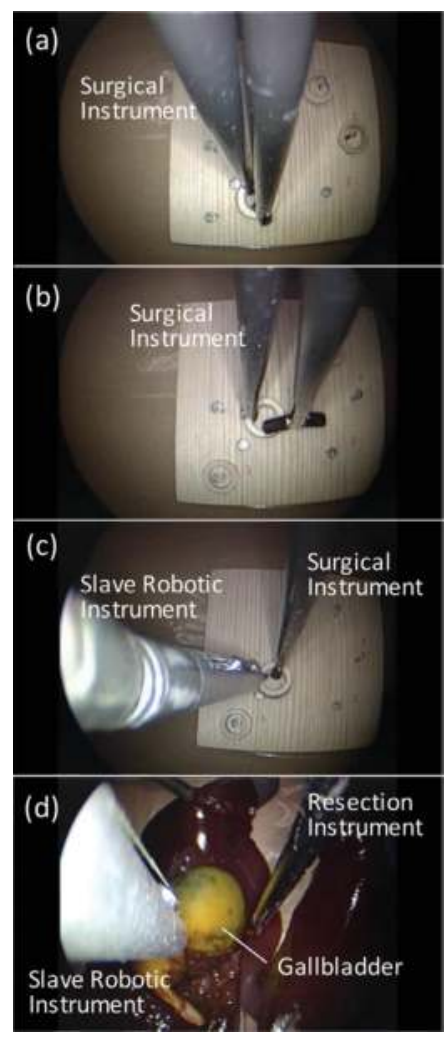

Figure 8. Laparoscopic view during the task; object-moving task in the operating condition of (a) SILSc, (b) SILSp, (c) proposed SILS, and (d) ex vivo cholecystectomy using our local-operated master-slave robot system. 


\section{Discussion}

As for the remote-operated master-slave robot system, from Experiment (1), we confirmed that the average object-moving time and standard deviation in the proposed SILS were significantly shorter than in iSPS, representing conventional single-port surgery, and were almost the same as those in iMPS, representing conventional multi-port surgery. From the subjective evaluations, all surgeons commented that the operability in the proposed SILS was little differentiated from that in iMPS. In Experiment (2), the object-touching task in the proposed SILS was accomplished in shorter time than iSPS, and there was less interference between the instruments in the proposed SILS than in iSPS. These results indicate that our proposed remote-operated system could improve the operability of SILS and achieve operability close to conventional multi-port surgery by adding two virtual incisions.

In regard to the local-operated master-slave robot system, in Experiment (1), the average switching time and standard deviation for proposed SILS were shorter than those for two conventional SILS operating conditions (SILSc and SILSp). Instances of interference between the surgical instruments were drastically decreased in the proposed SILS as compared with SILSc and SILSp. Focusing on the left-side surgical instrument, its location in the laparoscopic view in our proposed SILS differed from that of conventional SILS methods, as shown in Figure 8(a)-(c). The left-side and right-side instruments were inserted from the same direction on the laparoscopic view and were close together in SILSc and SILSp, and the left-side instrument was inserted from left side on the laparoscopic view as in conventional multi-port laparoscopic surgery in proposed SILS. The subjective evaluations from the surgeons also indicated that they were able to conduct SILS more easily using our proposed system than conventional SILS. In Experiment (2), the operator performed single-port laparoscopic cholecystectomy without any problems, and a laparoscopic view close to that in multi-port surgery was observed as shown in Figure 8(d). The operator also reported that it was like performing laparoscopic surgery though there was only one port. From these results, we confirmed that the local-operated master-slave robot system could improve the operability of SILS, providing greater efficiency and stability, and offering the operability and a surgical view similar to those of multi-port laparoscopic surgery by introducing one virtual incision.

From the above results of the two master-slave robot systems, we have confirmed the usefulness of our proposed new "virtual incision" surgical approach, since it brings the operability and surgical view similar to multi-port laparoscopic surgery even though only one port is opened in the abdominal wall. The operational procedure of our proposed surgical robot systems is close to the conventional surgical method, and thus does not require extra training for surgeons who are used to conventional multi-port laparoscopic surgery.

Although the initial motivation for proposing the "virtual incision" concept is to overcome the difficulties of SILS, such as instrument collision and visual defects, this concept can be used not only for single-port surgery but also for conventional multi-port surgery. For example, three-port surgery could be conducted with two real openings by adding one "virtual incision". In thoracoscopic surgery, the surgical port position is limited by the alignment of the limb, and is not as flexible as in laparoscopic surgery. By introducing the concept of "virtual 
incision" to thoracoscopic surgery, it is expected that surgeons can make a surgical port at an appropriate position without worrying about the limb, and the operating area can expand with fewer ports. We plan to develop a surgical device introducing our concept for thoracoscopic surgery [16].

Needlescopic surgery, another form of minimally invasive surgery, has been utilized in various surgical fields and brings cosmetic benefits for patients since it uses surgical instruments with very small diameter $[17,18]$; however, it also has problems as it is difficult to keep the stiffness of the surgical instrument while including adequate functionality in a small-diameter instrument. We believe that our new surgical concept of "virtual incision" is one way to achieve more minimally invasive surgery by reducing the number of real ports through a virtual incision.

As for master-slave surgical robot systems, many surgical robot systems have been developed to support surgery, but a master can control only a slave of the same system. For future robotic surgery, we think that collaboration among various master-slave surgical robot systems will become increasingly important. Operating surgeons have different preferences for the master controller of a system, and it is difficult to decide on one master. Robotic surgery could become more flexible if multiple masters can control the same slave or one slave can be easily exchanged for another. We are also working on another project in which multi-master and multi-slave options could be selected flexibly using an industrial middleware ORiN for a next-generation surgical-assisted robot system [19].

\section{Conclusion}

Minimally invasive approaches such as reduced port surgery have been introduced to various surgical fields to achieve more patient-friendly surgery. Although single-incision laparoscopic surgery (SILS) has cosmetic benefits for patients, such as less pain, smaller scar, and shorter hospitalization than conventional laparoscopic surgery due to its single opening, it has technical problems that increase the surgeon's burden. Therefore, we have proposed a new surgical approach called a "virtual incision", and have applied it to two types of master-slave robot systems for assisting SILS. From the evaluation results of the two master-slave robot systems, we have confirmed the effectiveness of "virtual incision" because the operability of SILS was improved by increasing the number of ports virtually. In this chapter, we have focused on SILS; however, our surgical approach could also be useful to multi-port surgery. We believe that the concept of "virtual incision" is a promising surgical approach that preserves QOL for both patients and surgeons.

\section{Acknowledgements}

This research was partly supported by MEXT KAKENHI Grant Number 15K16338 and 16H01 859. 


\section{Author details}

Yuki Horise ${ }^{1}$, Atsushi Nishikawa ${ }^{2 *}$, Toshikazu Kawai ${ }^{3}$, Ken $_{\text {Masamune }}{ }^{1}$ and Yoshihiro Muragaki ${ }^{1}$

*Address all correspondence to: nishikawa@shinshu-u.ac.jp

1 Faculty of Advanced Techno-Surgery, Tokyo Women's Medical University, Tokyo, Japan

2 Faculty of Textile Science and Technology, Shinshu University, Ueda, Japan

3 Faculty of Engineering, Osaka Institute of Technology, Osaka, Japan

\section{References}

[1] Lange R, Bleiziffer S, Mazzitelli D, Elhmidi Y, Opitz A, Krane M, Deutsch M-A, Ruge H, Brockmann G, Voss B, Schreiber C, Tassani P, Piazza N. Improvements in transcatheter aortic valve implantation outcomes in lower surgical risk patients: A glimpse into the future. Journal of the American College of Cardiology. 2012;59(3):280-287

[2] Grutters JPC, Kessels AGH, Pijls-Johannesma M, De Ruysscher D, Joore MA, Lambin P. Comparison of the effectiveness of radiotherapy with photons, protons and carbonions for non-small cell lung cancer: A meta-analysis. Radiotherapy and Oncology. 2010;95(1):32-40

[3] Lacy AM, García-Valdecasas JC, Delgado S, Castells A, Taurá P, Piqué JM, Visa J. Laparoscopy-assisted colectomy versus open colectomy for treatment of non-metastatic colon cancer: A randomized trial. The Lancet. 2002;359(9325):2224-2229

[4] Prasad A, Mukherjee KA, Kaul S, Kaur M. Postoperative pain after cholecystectomy: Conventional laparoscopy versus single-incision laparoscopic surgery. Minimally Access Surgery. 2011;7(1):24-27

[5] Desai MM, Rao PP, Aron M, Pascal-Haber G, Desai MR, Mishra S, Kaouk JH, Gill IS. Scarless single port transumbilical nephrectomy and pyeloplasty: First clinical report. Journal Compilation BJU International. 2008;101(1):83-88

[6] Ross S, Rosemurgy A, Albrink M, Choung E, Dapri G, Gallagher S, Hernandez J, Horgan S, Kelley W, Kia M, Marks J, Martinez J, Mintz Y, Oleynikov D, Pryor A, Rattner D, Rivas H, Roberts K, Rubach E, Schwaitzberg S, Swanstrom L, Sweeney J, Wilson E, Zemon H, Zundel N. Consensus statement of the consortium for LESS cholecystectomy. Surgical Endoscopy. 2012;26(10):2711-2766

[7] Autorino R, Kaouk JH, Stolzenburg J-U, Gill IS, Mottrie A, Tewari A, Cadeddu JA. Current status and future directions of robotic Single-Site surgery: A systematic review. European Urology. 2013;63(2):266-280 
[8] Romanelli JR, Earle DB. Single-port laparoscopic surgery: An overview. Surgical Endoscopy. 2009;23(7):1419-1427

[9] Mutter D, Callari C, Diana M, Dallemagne B, Leroy J, Marescaux J. Single port laparoscopic cholecystectomy: Which technique, which surgeon, for which patient? A study of the implementation in a teaching hospital. Journal of Hepato-Biliary-Pancreatic Sciences. 2011;18(3):453-457

[10] Ma J, Cassera MA, Spaun GO, Hammill CW, Hansen PD, Aliabadi-Wahle S. Randomized controlled trial comparing single-port laparoscopic cholecystectomy and four-port laparoscopic cholecystectomy. Annals of Surgery. 2011;254(1):22-27

[11] Erbella Jr J, Bunch GM. Single-incision laparoscopic cholecystectomy: The first 100 outpatients. Surgical Endoscopy. 2010;24:1958-1961

[12] Kroh M, El-Hayek K, Rosenblatt S, Chand B, Escobar P, Kaouk J, Chalikonda S. First human surgery with a novel single-port robotic system: Cholecystectomy using the da Vinci Single-Site platform. Surgical Endoscopy. 2011;25:3566-3573

[13] Park S-S, Kim M-C, Park MS, Hyung WJ. Rapid adaptation of robotic gastrectomy for gastric cancer by experienced laparoscopic surgeons. Surgical Endoscopy. 2012;26:60-67

[14] Horise Y, Nishikawa A, Sekimoto M, Kitanaka Y, Miyoshi N, Takiguchi S, Doki Y, Mori M, Miyazaki F. Development and evaluation of a master-slave robot system for single-incision laparoscopic surgery. International Journal of Computer Assisted Radiology and Surgery. 2012;7(2):289-296

[15] Horise Y, Matsumoto T, Ikeda H, Nakamura Y, Yamasaki M, Sawada G, Tsukao Y, Nakahara Y, Yamamoto M, Takiguchi S, Doki Y, Mori M, Miyazaki F, Sekimoto M, Kawai T, Nishikawa A. A novel locally operated master-slave robot system for singleincision laparoscopic surgery. Minimally Invasive Therapy \& Allied Technologies. 2014;23(6):326-332

[16] Horise Y, Nishikawa A, Kawai T, Kanzaki M, Masamune K, Muragaki Y. Endoscopic surgical support system with a concept of "Virtual Incision". Journal of Japan Society of Computer Aided Surgery. 2015;17(3):277-278

[17] Cheah WK, Lenzi JE, So JBY, Kum CK, Goh PMY. Randomized trial of needlescopic versus laparoscopic cholecystectomy. British Journal of Surgery. 2001;88(1):45-47

[18] Mamazza J, Schlachta CM, Seshadri PA, Cadeddu MO, Poulin EC. Needlescopic surgery. Surgical Endoscopy. 2001;15(10):1205-1212

[19] Okamoto J, Horise Y, Masamune K, Iseki H, Muragaki Y. Development of a prototype model of "Hyper SCOT (Smart Cyber Operating Theater)". International Journal of Computer Assisted Radiology and Surgery. 2016;11(Supplement 1):S163 
